\title{
Development of a Competency-based Training in Obstetrics and Gynecology Ultrasound for Undergraduate and Graduate Medical Education
}

1Dolgor Baatar, ${ }^{2}$ Jesus Peinado, ${ }^{3}$ Jorge Sarmiento, ${ }^{4} \mathrm{Hoi}$ Ho

\begin{abstract}
Advances in technology have made ultrasonography a rapidly evolving concept in the practice of medicine and a valuable component of the competency-based education. American Medical Association (AMA) recently affirms that 'ultrasound imaging is a safe, effective and efficient tool when utilized by, or under the direction of appropriately trained physicians.' AMA also supports the educational efforts and widespread integration of ultrasound throughout the continuum of medical education.

Training in ultrasonography is rapidly expanding to numerous residency programs of graduate medical education but discrepancies in ultrasound curriculum and criteria for proficiency exist among programs within the same discipline, despite clearly defined objectives recommended by the governing bodies. There is a trend to integrate ultrasonography into the curriculum of undergraduate medical education. However, funding, availability of ultrasound-trained faculty and student time are barriers to the implementation. Ultrasonography is a natural fit for competency-based training and should be introduced early in medical education. We expect that the LCME will soon mandate the integration of ultrasound into the 4-year curriculum.

The imminent question that medical educators ask is not when ultrasound will become a required component of the curriculum but how to effectively integrate the teaching and training of ultrasound into the continuum of medical education.
\end{abstract}

Keywords: Clinical competence, Competency-based education, Obstetrics and gynecology education, Internship and residency, Ultrasonography, Undergraduate medical education, Graduate medical education.

How to cite this article: Baatar D, Peinado J, Sarmiento J, Ho $\mathrm{H}$. Development of a Competency-based Training in Obstetrics

\footnotetext{
${ }^{1-3}$ Assistant Professor, ${ }^{4}$ Professor

${ }^{1}$ Department of Medical Education, Paul L Foster School of Medicine, Texas Tech University Health Sciences Center at EI Paso, Texas, USA

${ }^{2}$ Department of Pediatrics, Paul L Foster School of Medicine Texas Tech University Health Sciences Center at EI Paso Texas, USA

${ }^{3}$ Department of Radiology, Paul L Foster School of Medicine Texas Tech University Health Sciences Center at El Paso Texas, USA

${ }^{4}$ Department of Internal Medicine, Office of Faculty Affairs and Faculty Development, Paul L Foster School of Medicine, Texas Tech University Health Sciences Center at EI Paso, Texas, USA

Corresponding Author: Dolgor Baatar, Assistant Professor Department of Medical Education, Paul L Foster School of Medicine Texas Tech University Health Sciences Center, 5001 El Paso TX 79905, USA, e-mail: dolgor.baatar@ttuhsc.edu
}

and Gynecology Ultrasound for Undergraduate and Graduate Medical Education. Donald School J Ultrasound Obstet Gynecol 2014;8(1):83-86.

Source of support: Nil

Conflict of interest: None

\section{INTRODUCTION}

Ultrasonography is a proven valuable clinical tool with numerous applications in the diagnosis and management of different diseases across many specialties. It's quite obvious that during the last two decades, the ultrasound technology has outpaced the level of integration of ultrasound in the continuum of medical education, particularly the undergraduate medical education (UGME).

\section{ULTRASOUND IN UNDERGRADUATE MEDICAL EDUCATION}

The ultrasound training component of residency programs has dramatically expanded during the recent years. ${ }^{1}$ Although ultrasound curriculum for residents in obstetrics and gynecology has been developed and successfully implemented in several programs, ${ }^{2}$ the specific ultrasound curriculum in obstetrics and gynecology for medical students is yet to be developed. It should be mentioned that a large amount of the theoretical material and the long list of required competencies are significant challenges for both the residents and program directors. Accordingly, it will be a practical and valuable approach to reduce the educational burden for residency programs including ultrasound-related objectives and to transfer these materials to the curriculum of undergraduate years of medical school. Here we will discuss important objectives in obstetrics and gynecology ultrasound education that are directly linked to the competencies for obstetrics and gynecology residents but could be successfully integrated into the curriculum of several pre-clerkship disciplines. This transformation of the curriculum will assist the students to understand the pathophysiology of reproductive organs diseases and the mechanism of action of ultrasound technologies used in the prevention, diagnosis and treatment of these diseases.

\section{Physiology}

The International Society of Ultrasound in Obstetrics and Gynecology (ISUOG) Educational Committee developed 
and published minimum standards for ultrasound training for residents in obstetrics and gynecology. ${ }^{3}$ The theoretical training program included objectives on basic principles of medical ultrasound such as the relevant principles of acoustics, attenuation, absorption, reflection, speed of sound, the effects on tissues of pulsed and continuous wave ultrasound beam and the Doppler principle. We believe that these objectives can be successfully integrated into the physiology curriculum of the first year of the medical school.

\section{Anatomy and Pathology}

The Department of Obstetrics and Gynecology, University of New Mexico Health Sciences Center developed and has been using since 2006 a comprehensive curriculum in ultrasound education for obstetrics and gynecology residents. ${ }^{1}$ Analysis of this curriculum reveals several topics that are also important for medical students. These topics include objectives that are typically covered by the anatomy or pathology curriculum, such as the anatomy of female reproductive organs, embryo and fetus, cul-de-sac fluid, and uterine and/or adnexal masses.

Previous studies have demonstrated feasibility and effectiveness of the integration of focused sonography into the anatomy and pathophysiology curriculum. ${ }^{4,5}$ In view of the predominance of basic science disciplines in the preclinical years, basic ultrasound principles and image acquisition were covered during this time. Indications and interpretation of ultrasound were highlighted during the clinical years. ${ }^{6}$

To effectively incorporate the ultrasound objectives into the curriculum of anatomy and pathology of reproductive organs, embryo and fetus, in addition to the cross sectional, macro and microscopic images, we need to extensively utilize the ultrasound images and videos. Along with these changes, we need to train the basic science educators and/or include clinical faculty skillful in ultrasound imaging in the delivery of this innovative curriculum. It is expected that by the end of first two years the medical students will be able to evaluate normal ultrasound images of the reproductive organs and the fetus, as well as to recognize the common pathologies of the female reproductive organs and recognize sonoembryologic findings (Table 1).

\section{Medical Skills}

In 2006, the University of South Carolina, School of Medicine introduced an innovative integrated ultrasound curriculum (iUSC) across all 4 years ${ }^{7}$ in which obstetrics and gynecology ultrasound is taught during the 2nd and 3 rd years of the medical school. Transabdominal component of female pelvic ultrasound is taught as a laboratory session
Table 1: Proposed competency-based objectives for integrating ultrasound into the anatomy and pathology curriculum of the female reproductive organs during the preclinical years of the medical school

By the end of ultrasound integrated curriculum the learner will be able to recognize:

1 The anatomy of the female reproductive organs:

a. Recognize uterine and ovarian anatomy in sagittal and transverse planes

b. Correctly identify bladder, uterus, vagina, ovaries and posterior cul-de-sac

c. Correctly document sagittal and transverse images of iliac vessels and pelvic muscles

2. The anatomy of the embryo:

a. Correctly identify the yolk sac and extraembryonic mesoderm

b. Correctly identify, document and measure the gestational sac

c. Correctly identify, document and measure the crownrump length of the embryo

3. The anatomy of the fetus:

a. Correctly identify fetal lie and location of fetal spine and extremities

b. Be able to describe location and grade of the placenta

c. Be able to identify cord insertion in the anterior wall of fetal abdomen and at placenta

d. Be able to complete individual parameters of obstetric examination protocol with supervision, including:

i. Biparietal diameter

ii. Head circumference

iii. Four-chamber view of the fetal heart

iv. Abdominal circumference

v. Femur and humerus length

4. Pathology of the female reproductive organs:

a. Correctly document any pathologic findings in the pelvis, including cul-de-sac fluid or masses in the uterus or adnexa

with the use of standardized patients in conjunction with the 'Introduction to Clinical Medicine' course during the spring semester of the second year. Analysis of surveys indicated that students significantly benefited from the introduction of ultrasound teaching during the iUCS. The training of students on pregnant standardized patients would be very valuable, however, ethical issues are major concerns because several professional organizations in ultrasound have advised against performing fetal ultrasound exams that are not medically indicated. ${ }^{8}$ This is the area where the learners can gain experience with the practice on obstetrical ultrasound clinical simulators such as the VIMEDIX Women' Health which can provide an opportunity for comprehensive and realistic assessment of both normal and abnormal fetal anatomy, biometry and maternal adnexal anatomy.

Teaching basic ultrasound skills to clerkship students using focused assessment with sonography in trauma (FAST) has been widely accepted by the participants who 
uniformly believed that ultrasound training should be a regular component of the curriculum. ${ }^{9}$

Funding for equipment is the major challenge to the implementation of focused ultrasound education in the UGME curriculum. ${ }^{6}$ Obviously, as the prices of newer ultrasound scanners continue dropping, funding for the program will no longer remain the major issue. Other challenges to the program are availability of the clinical faculty and student time, given the constraint of heavy clinical load of the faculty and the student's schedule. ${ }^{6}$

The integration of ultrasonography into the 4-year curriculum of UGME will fulfill many Liaison Committee on Medical Education (LCME) criteria for accreditation including the Educational Program for MD degree ED-12: The curriculum of a medical education program should include laboratory or other practical opportunities for the direct application of the scientific method, accurate observation of biomedical phenomena and critical analysis of data. In view of positive results reported by medical schools where a vertical curriculum in ultrasound is implemented, we expect that the LCME will soon require an effective implementation of such curriculum. ${ }^{6}$

\section{ULTRASOUND IN GRADUATE MEDICAL EDUCATION}

Diagnostic ultrasonography has been widely used by physicians in clinical practice and in the public health sector in the last 50 years. Focused ultrasonography is an evolving concept in medicine and has been shown to be a valuable component of competency-based education. Ultrasonography is rapidly expanding to numerous residency programs of graduate medical education (GME) including radiology, obstetrics and gynecology, anesthesiology, emergency medicine, family medicine, internal medicine, neurology, pediatrics and surgery.

American Medical Association (AMA) recently affirms that 'ultrasound imaging is a safe, effective, and efficient tool when utilized by, or under the direction of appropriately trained physicians.' AMA also supports the educational efforts and widespread integration of ultrasound throughout the continuum of medical education.

Governing bodies for medical specialties and/or medical education such as the American College of Emergency Physician (ACEP) or the Accreditation Council for Graduate Medical Education (ACGME) defined the use of ultrasonography in many clinical conditions as well as standards for patient safety and quality of care involving the training and application of ultrasound in patient care. The American Institute of Ultrasound in Medicine (AIUM) also established training guidelines for physicians who evaluate and interpret diagnostic abdominal, obstetric and gynecologic ultrasound examinations. AIUM requires completion of an approved residency program, fellowship and/or postgraduate training that includes the equivalent of at least 3 months of diagnostic ultrasound training in the area(s) they practice, under the supervision of a qualified physician(s), during which the trainees have evidence of being involved with the performance, evaluation and interpretation of at least 300 sonograms. ${ }^{10}$ Currently the Society for Academic Emergency Medicine (SAEM) task force recommends 40 hours of instruction for training in ultrasound, a total of 150 examinations and that more than $50 \%$ of these examinations should be in those patients for whom it is clinically indicated. ${ }^{10}$ This short training program contrasts with those of other specialty groups where up to 100 hours of continuing professional development and 500 examinations are required. ${ }^{11}$ However, discrepancies in the ultrasound curriculum, quality of training and criteria for proficiency exist among residency programs within the same discipline. $^{12}$

Fellowships in diagnostic ultrasound in radiology and maternal fetal medicine have specific curricula; however, there are no standardized requirements or curriculum for residency training in obstetrics and gynecology. In 2009, AIUM published the guidelines for the performance of pelvic ultrasound examinations. ${ }^{13}$ Full-time teaching faculty and sonographers are important individuals contributing to the effective implementation of objective-structured and competency-based ultrasound curriculum training for obstetrics and gynecology residents. It is also essential to incorporate practice sessions on obstetrics and gynecology ultrasound clinical simulator to the required bedside training in ultrasound.

\section{CONCLUSION}

Ultrasonography is rapidly expanding in the practice of medicine across many specialties, and consequently, training in ultrasonography is incorporated into the numerous residency programs of graduate medical education. However, discrepancies in the ultrasound curriculum, quality of training and criteria for proficiency exist among programs within the same discipline. Preliminary results of the integration of a vertical curriculum in ultrasound in undergraduate medical education has been largely positive and we expect that the LCME will soon mandate the integration of ultrasound into the 4-year curriculum of the medical school.

\section{REFERENCES}

1. Hall R, Ogburn T, Rogers RG. Teaching and evaluating ultrasound skill attainment: competency-based resident ultrasound training for AIUM accreditation. Obstet Gynecol Clin North Am 2006;33(2):305-323. 
2. Lee $\mathrm{W}$ et al. Fetal ultrasound training for obstetrics and gynecology residents. Obstet Gynecol 2004;103(2):333-338

3. ISUOG education committee. Ultrasound in Obstetrics and Gynecology 1996;8(5):363-365.

4. Tshibwabwa ET, Groves HM. Integration of ultrasound in the education programme in anatomy. Med Educ 2005;39(11):1148.

5. Hoppmann RA, et al. An integrated ultrasound curriculum (iUSC) for medical students: four-year experience. Crit Ultrasound J 2011;3(1):1-12.

6. Bahner DP, et al. Integrated medical school ultrasound: development of an ultrasound vertical curriculum. Crit Ultrasound J 2013;5(1):6.

7. Hoppmann R, et al. Ultrasound in medical education: a vertical curriculum at the University of South Carolina School of Medicine. JSC Med Assoc 2006;102(10):330-334.
8. ISUOG Practice Guidelines: performance of first-trimester fetal ultrasound scan. Ultrasound in Obstetrics and Gynecology 2013;41(1):102-113.

9. Gogalniceanu P, et al. Is basic emergency ultrasound training feasible as part of standard undergraduate medical education? J Surg Educ 2010;67(3):152-156.

10. Training Guidelines for Physicians Who Evaluate and Interpret Diagnostic Abdominal, Obstetric, and/or Gynecologic Ultrasound Examinations. 2013; Available at: http://www.aium. org $/$ resources $/$ viewStatement.aspx?id $=47$.

11. Heller MB, et al. Residency training in emergency ultrasound: fulfilling the mandate. Acad Emerg Med 2002;9(8):835-839.

12. Ahern $\mathrm{M}$, et al. Variability in ultrasound education among emergency medicine residencies. West J Emerg Med 2010;11(4):314-318.

13. AIUM Practice Guideline for the Performance of Pelvic Ultrasound Examinations. 2009. Available at: http://www.aium. org/resources/guidelines/pelvic.pdf 